Res Publica. Revista de Historia de las Ideas Políticas

ISSN: $1576-4184$

http://dx.doi.org/10.5209/RPUB.56469

\title{
La dimensión moral de la Declaración de derechos de 1789: algunas reflexiones a partir de la teoría del reconocimiento Axel Honneth
}

\author{
Pablo Facundo Escalante*
}

Recibido: 12 de septiembre de 2016 / Aceptado: 10 de mayo de 2017

Resumen. Desde el momento mismo de su proclamación, la autoevidente naturaleza política de la Declaración de derechos del hombre y del ciudadano ha contribuido a opacar el carácter moral de los principios allí enunciados. A partir de la teoría del reconocimiento elaborada por Axel Honneth, este artículo intentará recuperar la dimensión moral de dicho documento. Con este propósito, el análisis aquí propuesto se concentrará no tanto en el propio texto de la Declaración, como en los debates parlamentarios que la produjeron.

Palabras clave: Revolución francesa; Derechos del hombre; Axel Honneth; teoría del reconocimiento; cultura política revolucionaria.

\section{[en] The Moral Dimension of the Declaration of Rights of 1789: Some Considerations based on Axel Honneth's Theory of Recognition}

Abstract. From the very moment of its proclamation, the self-evident political nature of the Declaration of the Rights of Man and of the Citizen aided to overshadow the moral character of its principles. Based on the theory of recognition developed by Axel Honneth, this paper will try to recover the moral dimension of said document. To this end, the analysis proposed here will focus not as much on the actual text of the Declaration as on the parliamentary debates that produced it.

Keywords: French revolution; Rights of man; Axel Honneth; theory of tecognition; revolutionary political culture.

El recuerdo de los males que se han padecido, de aquellos a los que se ha sido más sensible, dirige, en general, la pluma de los redactores de las declaraciones de derechos.

Sieyès ${ }^{1}$

Sumario: 1. El valor moral de los derechos a la luz de la teoría del reconocimiento. 2. Consagrando la igualdad de derechos, piedra angular en la formación del autorrespeto 3. El carácter universal de la Declaración. 4. Las consecuencias de la Declaración: el reconocimiento de la igualdad de derechos. 5. Los límites del reconocimiento

\footnotetext{
* Universidad Nacional de San Martín, Argentina

pf.escalante@yahoo.com.ar

1 C. Fauré (ed.), Des Manuscrits de Sieyès, 1773-1799, Paris, Honoré Champion, 1999, p. 499: “Le souvenir des maux qu'on a soufferts, de ceux auxquels on a été le plus sensible dirige en général la plume des rédacteurs des déclarations des droits".
} 
Cómo citar: Facundo Escalante, P. (2017). La dimensión moral de la Declaración de derechos de 1789: algunas reflexiones a partir de la teoría del conocimiento de Axel Honneth, en Res publica 20.2, 259-274.

Desde un primer momento, los diputados de la Asamblea nacional constituyente concibieron a la Declaración de los derechos del hombre y del ciudadano como una potente arma política destinada darle el golpe de gracia a un ya mortalmente herido Antiguo Régimen. "Antes de ser un texto-observa Marcel Gauchet-, la Declaración es un acto"2. En efecto, se trataba de un acto político que debía enterrar lo viejo para, simultáneamente, instituir lo nuevo. La naturaleza eminentemente política y fundacional aquel documento ofreció la arista más visible para quienes se interesaron por estudiarla y analizarla ${ }^{3}$. La inmediata y mordaz crítica que volcó Edmund Burke en sus Reflections on the Revolution in France, denunciando a la obra de la Constituyente como un agregado de "abstracciones metafísicas", es tan conocida como aquella realizada luego por Jeremy Bentham, quien la calificó como un "sinsentido sobre zancos", o como la que haría más tarde el propio Karl Marx, quien la definió como una "mónada aislada y replegada sobre sí misma"4. El hábito de abordar la Declaración desde una perspectiva filosófico-política ha conducido, sin embargo, a que se perdiera de vista el carácter profundamente moral de dicho documento.

En las páginas subsiguientes, se intentará, pues, recuperar la dimensión moral de la Declaración de derechos de 1789. Esta faceta, es preciso reconocerlo, no se percibe tan fácilmente a través del texto que compone sus diecisiete artículos. Como lo advirtió Sieyès en sus observaciones preliminares sobre la Constitución francesa, la exposición de las "grandes verdades" mediante la enumeración de una serie de principios no hace más que "suprimir de su contenido todo lo que exige la atención, todo lo que trasciende las cosas triviales que todo el mundo conoce por anticipado"s. Por consiguiente, el foco de la atención no se colocará tanto sobre el texto mismo de la Declaración, como sobre los debates parlamentarios en donde ella se originó, ya que, a través de los discursos de los diputados, es posible percibir con mayor claridad el carácter moral que ellos desearon imprimir sobre ese documento. Desde luego, para iluminar esta arista más recóndita de la Declaración, es preciso contar con las herramientas teóricas y metodológicas adecuadas. Afortunadamente, tales herramientas ya se encuentran a disposición de cualquier interesado, gracias al formidable trabajo realizado por Axel Honneth, y plasmado en su teoría del reconocimiento ${ }^{6}$.

M. Gauchet, La Révolution des droits de l'homme, Paris, Gallimard, 1989, p. 107: "Avant d'être un texte, la Déclaration est un acte".

3 Cf. C. Colliot-Thélène, "L'interprétation des droits de l'homme: enjeux politiques et théoriques au prisme du débat français", en Trivium 3, 2009, URL: http://trivium.revues.org/3290.

4 Cf. E. Burke, Reflexiones sobre la Revolución en Francia, Madrid, Alianza, [1790] 2003; J. Bentham, Tratado de los sofismas políticos, Buenos Aires, Leviatán, [1795] 2013; K. Marx, Sobre la cuestión judia, Buenos Aires, Prometeo Libros, [1844] 2004.

$5 \quad$ E.-J. Sieyès, Préliminaire de la Constitution. Reconnaissance et exposition raisonnée des Droits de l'Homme et du Citoyen. Lu les 20 et 21 Juillet 1789, au Comité de Constitution, Paris, Baudouin, 1789, p. 2: "retrancher de son sujet tout ce qui exige de l'attention, tout ce qui sort des choses triviales que tout le monde fait d'avance".

6 Cf. A. Honneth, La lucha por el reconocimiento. Por una gramática moral de los conflictos sociales, Barcelona, Crítica [1992] 1997. 


\section{El valor moral de los derechos a la luz de la teoría del reconocimiento}

A partir de la segunda mitad del siglo XX, varios autores han reflexionado alrededor del rol que las emociones poseen en el seno de la teoría social, entendiéndolas como

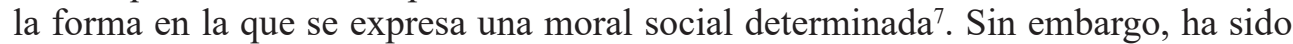
mérito exclusivo de Axel Honneth el haber desarrollado una teoría normativa de los conflictos sociales capaz de comprender e integrar la dimensión moral que, en mayor o menor medida, subyace detrás todos ellos.

[E]1 sufrimiento y el descontento sociales—subraya Honneth—poseen un núcleo normativo. Se trata de la frustración o violación de las expectativas normativas de la sociedad que consideren justificadas los afectados. Por tanto, esas sensaciones de descontento y de sufrimiento, en la medida en se califiquen como "sociales", coinciden con la experiencia de que la sociedad está haciendo algo injusto, algo injustificable ${ }^{8}$.

Ciertamente, para este pensador de la llamada tercera generación de la Escuela de Frankfurt, no caben dudas de que existe un vínculo emocional y, por lo tanto, moral entre, por un lado, el descontento y el sufrimiento sociales y, por otro lado, la experiencia misma de la injusticia social, cuyo origen, en última instancia, se encuentra en la ausencia de reconocimiento social que afecta a ciertos grupos o individuos. Se trata de una idea tomada de los primeros escritos de Hegel, anteriores a la Phänomenologie des Geistes, en donde este filósofo sostenía que la plena realización del ser humano se adquiere mediante relaciones éticas bien establecidas que implican el reconocimiento recíproco entre las personas involucradas, entendidas éstas como individuos con iguales derechos y capacidades. Lograr ese estado de reconocimiento recíproco requiere, no obstante, atravesar un proceso de desarrollo individual que se lleva adelante mediante el conflicto intersubjetivo, proceso al cual el propio Hegel denominó "lucha por el reconocimiento".

Sobre el surco que abrieron aquellas reflexiones hegelianas, Honneth extendió las propias, señalando precisamente que

la reproducción de la vida social se cumple bajo el imperativo de un reconocimiento recíproco, ya que los sujetos sólo pueden acceder a una autorrelación práctica si aprenden a concebirse a partir de la perspectiva normativa de sus compañeros de interacción, en tanto que sus destinatarios sociales?.

En efecto, aquel imperativo social "actúa como una coerción normativa que paulatinamente fuerza a los individuos a la delimitación del contenido de su reconocimiento recíproco, porque sólo por ello pueden conceder expresión social a las siem-

\footnotetext{
Cf., entre otros, E. Cahn, The Sense of Injustice: An Anthropocentric View of Law, New York, New York University Press, 1949; B. Moore Jr., Injustice: The Social Bases of Obedience and Revolt, New York, M. E. Sharpe, 1978; R. C. Solomon, A Passion for Justice: Emotions and the Origins of the Social Contract, Reading, Adisson-Wesley, 1990.

8 A. Honneth, "Redistribución como reconocimiento. Respuesta a Nancy Fraser", en N. Fraser y A. Honneth, ¿Redistribución o reconocimiento? Un debate político-filosófico, Madrid, Morata [2003] 2006, pp. 89-148, aquí p. 103.

$9 \quad$ A. Honneth, La lucha por el reconocimiento, op. cit., p. 114.
} 
pre crecientes pretensiones de su subjetividad"10. De hecho, a pesar de las críticas que suscitaron sus planteos iniciales ${ }^{11}$, Honneth ha sido capaz de demostrar que "incluso las injusticias distributivas deben entenderse como la expresión institucional de la falta de respeto social o, mejor dicho, de unas relaciones de reconocimiento injustificadas"12.

Preocupado por superar el carácter puramente metafísico de la propuesta hegeliana, Honneth procuró otorgarle un respaldo empírico a su teoría, recurriendo, para ello, al trabajo realizado por George Herbert Mead en el campo psicológico y sociológico. Las investigaciones de este psicólogo social condujeron a Honneth a percatarse de que "las reacciones de sentimientos negativos que psíquicamente acompañan la experiencia de menosprecio pueden presentar la base afectiva de impulsos en los que enraíza motivacionalmente la lucha por el reconocimiento"13. Es precisamente de este modo que se establece un nexo emocional y moral entre el descontento social y la ausencia de reconocimiento.

Al desarrollar su teoría, Honneth elaboró un esquema tripartito para explicar la autorrealización práctica del sujeto que se lleva a cabo mediante la lucha por el reconocimiento. En dicho esquema, la plena autorrealización se logra una vez que el individuo ha sido capaz de adquirir los sentimientos de autoconfianza, autorrespeto y autoestima. Ahora bien, Honneth señala que el autorrespeto, en particular, es un sentimiento privativo de la esfera legal, el cual se obtiene por medio del reconocimiento social de iguales derechos para todos los miembros de una misma comunidad. La igualdad de derechos adquiere así un valor moral para el sujeto, ya que "Sólo desde la perspectiva normativa de un «otro generalizado» podemos entendernos como personas de derecho, en el sentido que podemos estar seguros de la realización social de determinadas de nuestras pretensiones"14. "El carácter público de los derechos-observa Honneth-es aquello por lo que ellos autorizan a su portador a una acción perceptible por sus compañeros de interacción, lo que les concede la fuerza de posibilitar la formación del autorrespeto" ${ }^{15}$. Al verse revestidos con los mismos derechos, los individuos entienden que son reconocidos por el resto de la comunidad como personas autónomas y moralmente responsables, cuyos reclamos, al ampararse en una norma socialmente aceptada, les permite sentirse respetados por la sociedad ${ }^{16}$. Es por este motivo que, como lo advierte Joel Anderson, "las luchas sociales por cualquier tipo de expansión están orientadas hacia ideas de universalidad y autolegislación que hacen que sea normativamente ilegítimo (aunque quizás fácticamente acertado) el ver a los derechos como la encarnación de los intereses de clase"17.

La función fundamental que Honneth le atribuyó a los derechos en el proceso de formación del autorrespeto $-\mathrm{y}$, en última instancia, en la autorrealización práctica

\footnotetext{
Ibidem, pp. 114-115.

11 Cf. N. Fraser, "La justicia social en la era de la política de la identidad: Redistribución, reconocimiento y participación", en N. Fraser y A. Honneth, op. cit., pp. 17-88.

12 A. Honneth, "Redistribución como reconocimiento. Respuesta a Nancy Fraser", op. cit., p. 92.

13 A. Honneth, La lucha por el reconocimiento, op. cit., p. 165.

14 Ibidem, p. 133.

15 Ibidem, p. 147.

16 Cf. Ibidem. pp. 132-148.

17 J. Anderson, “Translator's Introduction”, en A. Honneth, The Struggle for Recognition. The Moral Grammar of Social Conflicts, Cambridge, Polity Press, [1992] 1995, pp. x-xxi, aquí p. xvi: "social struggles for either type of expansion are oriented to ideas of universality and self-legislation that make it normatively illegitimate (though perhaps factually accurate) to view rights as the embodiment of class interests".
} 
del sujeto en el seno de la lucha por el reconocimiento- es precisamente lo que permite recuperar la dimensión moral de la Declaración de los derechos del hombre y del ciudadano. En efecto, la pretendida universalidad de los principios enarbolados por ella estaba llamada a infundir el sentimiento de autorrespeto en la humanidad en su conjunto, ya que, como lo advierte Jean-Claude Milner, los derechos allí consagrados no estaban condicionados por la pertenencia a la comunidad política, debiendo proteger también a quienes se encontraban fuera de ella ${ }^{18}$. En su origen, pues, la Declaración estaba destinada a detener las injusticias y los abusos del Antiguo Régimen, tanto en Francia, como, eventualmente, en el resto del mundo.

\section{Consagrando la igualdad de derechos, piedra angular en la formación del autorrespeto}

En Francia, la segunda mitad del siglo XVIII estuvo caracterizada por un fuerte incremento de las denuncias contra los abusos del gobierno, especialmente contra aquellos que fueron señalados como una ofensa hacia la dignidad humana. En ese contexto, la experiencia cotidiana de la injusticia contribuyó a poner en circulación las nociones de derechos del hombre e igualdad de derechos, ambas concebidas como conceptos de combate [Kampfbegriffes] con los que se pretendía hacer frente a los vejámenes gubernamentales ${ }^{19}$. En el marco de aquella cruzada ilustrada contra el "despotismo", el concepto de igualdad de derechos en particular, no fue esgrimido de forma cínica, como si se tratara de una igualdad apenas teórica. Por el contrario, fue blandido como un verdadero concepto de ejercicio ${ }^{20}$.

Todas las personas—advierte Lynn Hunt—tendrían derechos humanos únicamente si todas ellas eran vistas como iguales de algún modo fundamental. La igualdad no era simplemente un concepto abstracto o una consigna política. Había de ser interiorizada de algún modo ${ }^{21}$.

Aquella concepción de la igualdad se encuentra perfectamente expresada en la Encyclopédie. Allí, bajo la voz "Igualdad natural", el caballero de Jaucourt aseguraba que como "la naturaleza humana es la misma en todos los hombres, está claro que según el derecho natural, cada uno debe estimar y tratar a los otros como seres que le son naturalmente iguales, es decir que son tan hombres como uno"22. Es precisamente esta concepción de la igualdad la que compartían los diputados del sexto gabinete de la Asamblea nacional constituyente. En efecto, el séptimo

18 Cf. J.-C. Milner, Relire la Révolution, Lagrasse, Verdier, 2016, pp. 169-216.

19 Sobre la noción de concepto de combate [Kampfbegriffe], cf. R. Koselleck, "A Response to Comments on the Geschichtliche Grundbegriffe", en H. Lehmann y M. Richter (eds.), The Meaning of Historical Terms and Concepts: New Studies on Begriffsgeschichte, Washington D.C., German Historical Institute, 1996, pp. 59-70.

20 Sobre la noción de concepto de ejercicio, cf. Ch. Taylor, “¿Cuál es el problema con la libertad negativa?”, en $L a$ libertad de los modernos, Buenos Aires, Amorrortu, 2005, pp. 257-281.

21 L. Hunt, La invención de los derechos humanos, Barcelona, Tusquets, [2007] 2009, p. 26.

22 L. de Jaucourt, "Égalité naturelle", en D. Diderot y J. le Rond d'Alembert (eds.), Encyclopédie ou dictionnaire raisonné des sciences, des arts et des métiers, t. V, Paris, 1755, p. 415: "Puisque la nature humaine se trouve la même dans tous les hommes, il est clair que selon le droit naturel, chacun doit estimer et traiter les autres comme autant d'êtres qui lui sont naturellement égaux, c'est-à-dire qui sont hommes aussi bien que lui'. 
artículo de su Proyecto de declaración de derechos del hombre y del ciudadano sostenía lo siguiente: "En el estado de sociedad, cada hombre, para obtener el ejercicio libre y legítimo de sus facultades, debe reconocerlo en sus semejantes, respetarlo y facilitarlo"23.

Claramente, el proyecto del sexto gabinete fundaba la igualdad de derechos bajo el imperativo moral de un reconocimiento recíproco de tales derechos. Aunque la redacción final de aquel principio terminó por ser mucho más lacónica que la propuesta del sexto gabinete ("Los hombres nacen y permanecen libres e iguales en derechos" ${ }^{24}$ ), el imperativo moral de reconocimiento recíproco de los derechos no desapareció de su espíritu. En efecto, Rabaut Saint-Étienne se valió de él para exigir a la Asamblea que el derecho a la libertad de culto quedara plasmado en la Declaración:

Señores, regreso a mis principios, o más bien a vuestros principios, ya que ellos os pertenecen: vosotros los habéis conquistado con vuestro coraje, y vosotros los habéis consagrado ante el mundo, declarando que todos los hombres nacen y permanecen libres e iguales. Los derechos de todos los franceses son los mismos, todos los franceses son iguales en derechos. No veo entonces ninguna razón para que una parte de los ciudadanos diga a la otra: yo seré libre, pero vosotros no lo seréis ${ }^{25}$.

Consecuente con el principio de igualdad natural de derechos que había sido establecido en el primer artículo de la Declaración, la Asamblea terminó por inscribir la libertad de culto en su artículo décimo: "Nadie debe ser inquietado por sus opiniones, ni siquiera por las religiosas, siempre que su manifestación no perturbe el orden público establecido por la ley"26. Fundado sobre el imperativo de reconocimiento recíproco, el artículo primero fue concebido para poner fin a aquello que moralmente era considerado como una de las mayores injusticias del Antiguo Régimen: la desigualdad jurídica y el privilegio. Precisamente, la originalidad de la Declaración, como lo advierte Marcel Gauchet, consistió en "proponer más bien una democratización del universo jerárquico que una simple sustitución de un sistema de normas por otro" 27 .

23 "Projet de déclaration des droits de l'homme et du citoyen, discuté dans le sixième bureau de l'Assemblée national (1789)", art. 7, en J. Mavidal y É. Laurent (eds.), Archives parlementaires de 1787 à 1860. Recueil complet des débats législatifs \& politiques des Chambres françaises, Première Série (1787 à 1799), t. VIII, Paris, Paul Dupont, 1875 (en adelante: $A P$ ), pp. 431-432, aquí p. 432: “Dans l'état de société, chaque homme, pour obtenir l'exercice libre et légitime de ses facultés, doit le reconnaître dans ses semblables, le respecter et le faciliter".

24 Declaración de los derechos del hombre y del ciudadano de 1789, art. 1.

25 Rabaut Saint-Etienne, 23 de agosto de 1789, en $A P$, t. VIII, p. 479: "Messieurs, je reviens à mes principes, ou plutôt à vos principes; car ils sont à vous: vous les avez conquis par votre courage, et vous les avez consacrés à la face du monde, en déclarant que tous les hommes naissent et demeurent libres et égaux. Les droits de tous les Français sont les mêmes, tous les Français sont égaux en droits. Je ne vois donc aucune raison pour qu'une partie des citoyens dise à l'autre: je serai libre, mais vous ne le serez pas".

26 Declaración de los derechos del hombre y del ciudadano de 1789, art. 10. El subrayado es mío.

27 M. Gauchet, op. cit., p. 114: “proposer plutôt une démocratisation de l'univers hiérarchique qu'une simple substitution d'un système de normes à l'autre". 


\section{El carácter universal de la Declaración}

El peso del principio de la igualdad natural de derechos consagrado en la Declaración se vio sin duda potenciado por el carácter universal y universalizante que los diputados plasmaron en ella. Como bien observó Lynn Hunt, "Las referencias a «los hombres», «el hombre», «cada hombre», «todo hombre», «todos los ciudadanos», «todo ciudadano», «la sociedad», y «toda sociedad» empequeñecían la referencia al pueblo francés" ${ }^{\prime 28}$. En efecto, en la opinión de los diputados, la Revolución había colocado a los franceses en una posición de privilegio con respecto a las otras naciones, en la medida en que los había convertido en un pueblo libre. Es por ese motivo que sentían la obligación de demostrar al mundo entero que el Nuevo Régimen era moralmente superior al Antiguo. "Sigamos el ejemplo de los Estados Unidos-declaró Montmorency-; ellos le dieron un gran ejemplo al nuevo hemisferio; démoslo nosotros al universo; presentémosle un modelo digno de ser admirado" 29 . Cierto, en la Declaración se jugaba también la legitimidad de la propia acción revolucionaria ${ }^{30}$. Sin embargo, no se puede negar la firme convicción — típica del pensamiento iluminista - que poseían los diputados, según la cual, una vez que una verdad ha sido revelada, ya no es posible presentarle oposición alguna. "Una declaración de derechos—señaló Mirabeau—, si pudiese responder a una perfección ideal, sería aquella que contuviese unos axiomas tan simples, evidentes y fecundos en consecuencias, que sería imposible desestimarla sin caer en el absurdo"31.

Los derechos contemplados en la Declaración pertenecían tanto al "ciudadano", como al "hombre" - es decir, al "no-ciudadano"-, de modo que las verdades que ellos expresaban no podían verse limitadas por la geografía. "No se trata aquí de hacer una declaración de derechos para Francia, sino para el hombre en general-aseguró Pétion-. Estos derechos no son leyes; estos derechos son de todos los tiempos y previos a las leyes" 32 . A decir verdad, esta pretensión universalista estuvo presente desde los primeros debates, cuando aún no se trataba más que de evaluar si era conveniente que una declaración de derechos antecediera a la Constitución. Ya por aquel entonces, el duque de Montmorency no dudó en sentenciar que "Los derechos del hombre son invariables como la justicia, eternos como la razón; son de todos los países y de todos los tiempos" ${ }^{\prime 3}$. Sus palabras pronto se convirtieron en una suerte de divisa con la cual los diputados procuraron expresar la validez universal que debían poseer los derechos incluidos en la Declaración. "[E]stos derechos —expresó Desmeuniers- son de todos los tiempos y de todas las naciones; han sobrevivido a los imperios en donde causaron dicha, y parecen participar de la eternidad de quien

28 L. Hunt, op. cit., p. 14.

29 Montmorency, $1^{\circ}$ de agosto de 1789, en $A P$, t. VIII, p. 320: "Suivons l'exemple des États-Unis; ils ont donné un grand exemple au nouvel hémisphère; donnons-le à l'univers; présentons-lui un modèle digne d'être admiré".

30 Cf. L. Cornu, Une autre république. 1791, L'occasion et le destin d'une initiative républicaine, Paris, L'Harmattan, 2004, pp. 167-185.

31 Mirabeau, 17 de agosto de 1789, en $A P$, t. VIII, p. 438: "Une déclaration des droits, si elle pouvait répondre à une perfection idéale, serait celle qui contiendrait des axiomes tellement simples, évidents et féconds en conséquences, qu'il serait impossible de s'en écarter sans être absurde".

32 Pétion, 23 de agosto de 1789, en $A P$, t. VIII, p. 475: "Il ne s'agit pas ici de faire une déclaration des droits seulement pour la France, mais pour l'homme en général. Ces droits ne sont pas des lois, et ces droits sont de tous les temps et avant les lois".

33 Montmorency, $1^{\circ}$ de agosto de 1789 , en $A P$, t. VIII, p. 320: "Les droits de l'homme sont invariables comme la justice, éternels comme la raison; ils sont de tous les temps et de tous les pays". 
los ha dictado" 34 . "No se trata aquí —observó Duport- de transigir con las circunstancias; no hay que temer aquí el decir las verdades de todos los tiempos y de todos los países"35. "Una declaración — señaló Duquesnoy- debe ser de todos los tiempos y de todos los pueblos; las circunstancias cambian, pero ella debe ser invariable en medio de las revoluciones" ${ }^{\prime 3}$.

Aquella pretensión universalista condujo rápidamente a los diputados a plantearse una declaración de derechos que fuera ejemplar. En ocasión de la presentación del proyecto que había sido encomendado al Comité de los cinco, Mirabeau exhortó a la Asamblea a redactar un documento que inspirara al resto del mundo:

vuestras leyes se volverán las de Europa, si ellas son dignas de vosotros: pues tal es la influencia de los grandes Estados, y sobre todo del imperio francés, en donde cada progreso en su constitución, en sus leyes, en su gobierno, agranda la razón y la perfectibilidad humana. [...] Es para nosotros, es para nuestros descendientes, es para el mundo entero que trabajáis vosotros; caminareis con un paso firme pero seguro hacia la gran obra ${ }^{37}$.

Una semana más tarde, durante los debates alrededor del derecho a la libertad de culto, Rabaut Saint-Étienne volvería a insistir sobre el carácter ejemplar que debía adquirir la Declaración:

Vosotros no estáis hechos para recibir el ejemplo, sino para darlo; el que existan pueblos injustos no implica que vosotros debáis serlo. Europa, que aspira a la libertad, espera de vosotros grandes lecciones, y vosotros sois dignos de otorgárselas. Que este código que vais a crear sea el modelo de todos los otros y que no quede en él ninguna tacha ${ }^{38}$.

Tal y como observa Keith Michael Baker, "La propia publicidad era la esencia de una declaración de derechos, la propia publicidad era la fuerza que habría de convertir semejante declaración en el parámetro de todos los gobiernos y en la piedra de toque de todas las leyes" ${ }^{\prime 3}$.

34 Desmeuniers, 3 de agosto de 1789, en $A P$, t. VIII, p. 334: "ces droits sont de tous les temps et de toutes les nations; ils ont survécu aux empires dont ils ont fait le bonheur, et ils semblent participer à l'éternité de celui qui les a dicté".

35 Duport, 18 de agosto de 1789, en $A P$, t. VIII, p. 451: "Il ne s'agit pas ici de composer avec les circonstances; il ne faut pas craindre ici de dire des vérités de tous les temps et de tous les pays".

36 Duquesnoy, 20 de agosto de 1789, en $A P$, t. VIII, p. 462: "Une déclaration doit être de tous les temps et de tous les peuples; les circonstances changent, mais elle doit être invariable au milieu des révolutions".

37 Mirabeau, 17 de agosto de 1789, en AP, t. VIII, p. 439: "vos lois deviendront celles de l'Europe, si elles sont dignes de vous: car telle est l'influence des grands États, et surtout de l'empire français, que chaque progrès dans leur constitution, dans leur lois, dans leur gouvernement, agrandit la raison et la perfectibilité humaine. [...] C'est pour nous, c'est pour nos neveux, c'est pour le monde entier que vous travaillez; vous marcherez d'un pas ferme mais mesuré vers le grand œuvre".

38 Rabaut Saint-Etienne, 23 de agosto de 1789, en AP, t. VIII, p. 479: "Vous n'êtes pas faits pour recevoir l'exemple, mais pour le donner; et de ce qu'il est des peuples injustes, il ne s'ensuit pas que vous deviez l'être. L'Europe, qui aspire à la liberté, attend de vous de grandes leçons, et vous êtes dignes de lui donner. Que ce code que vous allez former soit le modèle de tous les autres, et qu'il n'y reste aucune tache".

39 K. M. Baker, "The Idea of a Declaration of Rights", en D. Van Kley (ed.), The French Idea of Freedom: The Old Regime and the Declaration of Rights of 1789, Stanford, Stanford University Press, 1994, pp. 154-196, aquí p. 165: "Publicity itself was the essence of a declaration of rights, publicity itself was the force that would make such a declaration the measure of all governments and the touchstone of all laws". 
El universalismo era una de las características fundamentales de la teoría iusnaturalista que se había desarrollado a partir de mediados del siglo XVII y que se extendió por toda Europa occidental y América del Norte a lo largo del siglo XVIII ${ }^{40}$. El lenguaje de los derechos del hombre se había elaborado precisamente a partir de la concepción universalista del iusnaturalismo. Con todo, la euforia revolucionaria dio un impulso inesperado al universalismo iusnaturalista, en la medida en que los diputados - casi una cuarta parte de ellos formados en Derecho ${ }^{41}$ - comprendieron que la Revolución les había otorgado una oportunidad única para que los derechos del hombre dejaran de ser un enunciado teórico y pasaran a fundar un nuevo orden moral y político. "Todo debía cambiar con la revolución de Francia—recordaría Garat, años más tarde-[...]: en 1789, yo comencé a creer que las ideas, que hasta entonces me habían parecido apenas una bella quimera, podían realizarse sobre la tierra"42. Este optimismo se vio favorecido, además, por el hecho de que las deliberaciones sobre la declaración de derechos se llevaron a cabo en los primeros meses de la Revolución, de modo que los diputados aún no habían tenido ocasión de enfrentarse con las dificultades de legislar para una realidad social tan compleja como la que poseía Francia ${ }^{43}$. Habría que agregar, por otra parte, que aquel estado de optimismo pudo mantenerse en un nivel más o menos constante a causa de plazo relativamente breve en el que se llevaron a cabo aquellas deliberaciones, lo cual contribuyó a que los diputados no se distrajeran ni se afligieran por otros problemas emergentes ${ }^{44}$.

40 Para una breve exposición del pensamiento iusnaturalista, cf. N. Bobbio, "El modelo iusnaturalista", en N. Bobbio y M. Bovero, Sociedad y Estado en la filosofía politica moderna. El modelo iusnaturalista y el modelo hegeliano-marxiano, México, Fondo de Cultura Económica, [1979] 1986, pp. 13-145. Para la diseminación de las ideas iusnaturalistas, cf. L. Hunt, op. cit.

${ }^{41}$ Cf. E.-H. Lemay, "La composition de l'Assemblée nationale constituante: les hommes de la continuité?", Revue d'histoire moderne et contemporaine 3, 1977, pp. 341-363.

42 D.-J. Garat, Mémoires sur la Révolution, ou exposé de ma conduite dans les affaires et dans les fonctions publiques, Paris, J. J. Smits et C $C^{\text {ie }}, 1795$, p. 211: “Tout devait changer avec la révolution de France [...]: je commençai à espérer en 1789 , que des idées, qui ne m'avaient guère occupé jusqu'alors, que comme beau idéal, pourraient se réaliser sur la terre",

43 "[N]inguna educación—confesaría luego el conde de Clermont-Tonnerre-me había preparado para esta augusta función de redactar las leyes, función para la que Rousseau exigía una inteligencia superior capaz de contemplar todas las pasiones de los hombres y no verse afectada por ninguna; yo me equivoqué de buena fe, pero me equivoqué, y me hacían falta dos grandes maestros cuyas lecciones son a veces muy preciadas, el tiempo y el conocimiento de los hombres" (S. de Clermont-Tonnerre, Analyse raisonnée de la Constitution française, Paris, 1791, pp. 70-71: "aucune éducation ne m'avait préparé à cette auguste fonction de rédiger les lois, fonction pour laquelle Rousseau voulait une intelligence supérieure qui vît toutes les passions des hommes et n'en éprouvât aucune; je me trompais de bonne foi, mais je me trompais, et il me manquait deux grands maîtres, deux maitres dont les leçons sont quelquefois bien chères, le temps et la connaissance des hommes").

44 Partiendo de la propuesta del Comité de constitución, realizada el 9 de julio, la cronología de las deliberaciones sobre la Declaración se divide en dos momentos y tres instancias. Un primer momento, entre el $1^{\circ} \mathrm{y}$ el $4 \mathrm{de}$ agosto, constituye a su vez la primera instancia: en él se discutió la posibilidad de incluir la declaración de derechos a la cabeza de la Constitución. Un segundo momento es el que tuvo lugar entre el 18 y el 26 de agosto, en el cual, como segunda instancia (18 y 19) se debatió el proyecto del Comité de los cinco (formado el 12 de agosto por moción de Desmeuniers, con el propósito de evitar la discusión plenaria de cada uno de los proyectos presentados). La disconformidad con la propuesta de este comité llevó a que los diputados escogieran uno de los múltiples proyectos presentados (ganando el del sexto gabinete) y lo discutieran artículo por artículo en una tercera instancia ( 20 al 26 de agosto). Si bien en algunas sesiones de julio y agosto, ciertos diputados opinaron sobre el tema, se trató en todo momento de intervenciones aisladas; y si se toma en consideración que tanto el 2 como el 25 de agosto no hubo sesiones (el 2 fue domingo y el 25 la fiesta de San Luis), se debe concluir que las deliberaciones y la aprobación de la Declaración tuvieron lugar en apenas once días, lo cual es poco tiempo para una obra de semejante magnitud. 
La brevedad y la exclusividad de los debates alrededor de la declaración de derechos, junto al estado de ánimo optimista de los diputados, condujo a que tales deliberaciones se desarrollaran virtualmente en un tiempo suspendido. En efecto, en comparación con los demás debates en los que se vio sumida la Asamblea, los factores coyunturales ajenos a los diputados no intervinieron en aquellos vinculados a la declaración de derechos ${ }^{45}$. Esta ausencia de presiones externas infundió tanto optimismo en la Asamblea que los diputados se convencieron de que todo era posible y de que los únicos límites para la Declaración eran la Razón y la Justicia ${ }^{46}$. Esta convicción se puso particularmente en evidencia en la decisión de no acompañar los derechos incluidos en la Declaración con una lista de deberes. Durante la sesión del 4 de agosto, Grégoire había planteado precisamente la necesidad de elaborarla: "Es más que esencial hacer una declaración de deberes para sujetar a los hombres en los límites de sus derechos [...]. Hay que establecer un equilibrio, hay que mostrar al hombre el círculo que puede recorrer, y las barreras que pueden y deben detenerlo" 47 . Clermont-Lodève respondería entonces que el "término de ciudadano anuncia una correlación con los otros ciudadanos, y esa correlación engendra los deberes" ${ }^{\prime 4}$. La polémica que levantó esta cuestión entre los diputados condujo a que el presidente de la Asamblea terminara sometiéndola a votación: la inclusión de deberes fue rechazada por 570 votos en contra frente a 433 a favor ${ }^{49}$.

\section{Las consecuencias de la Declaración: el reconocimiento de la igualdad de derechos}

No caben dudas de que el lenguaje utilizado por los diputados en el curso de los debates, y plasmado luego en la propia Declaración, causó una descomunal apertura del horizonte de expectativa de los franceses ${ }^{50}$. Como lo advirtió Marcel Gauchet,

45 La interrupción de los debates en el artículo decimoséptimo y la decisión de postergar las deliberaciones hasta terminar con la Constitución fueron dos resoluciones vinculadas con la urgencia por finalizar la obra constitucional, urgencia que surgió en el propio seno de la Asamblea. Sin duda, los plazos de la Asamblea estaban ligados a factores externos. No obstante, a lo largo de los debates sobre la declaración de derechos, no ocurrió ningún acontecimiento tan importante como para ejercer presión en los diputados de forma colectiva, a diferencia de lo que había sucedido, por ejemplo, el 4 de agosto, fecha en la que la abolición del "régimen feudal" fue decretada con el propósito apaciguar el malestar social que había sido provocado por el "gran miedo".

46 "La fuerza, la ambición, la ignorancia han formado y sostienen aún a la mayoría de los gobiernos-observó Romme-: se querría fundar el nuestro sobre la razón y la justicia como los únicos garantes de la libertad y de los derechos naturales del hombre" (A. Galante Garrone, Gilbert Romme. Histoire d'un révolutionnaire (17501795), Paris, Flammarion, 1971, p. 178: “La force, l'ambition, l'ignorance ont formé et maintiennent encore la plupart des gouvernements: on voudrait fonder le nôtre sur la raison et la justice comme les seuls garants de la liberté et des droits naturels de l'homme").

47 Grégoire, 4 de agosto de 1789, en $A P$, t. VIII, p. 340: "Il est principalement essentiel de faire une déclaration des devoirs pour retenir les hommes dans les limites de leurs droits [...]. Il faut établir un équilibre, il faut montre à l'homme le cercle qu'il peut parcourir, et les barrières qui peuvent et doivent l'arrêter".

48 Clermont-Lodève, 4 de agosto de 1789, en AP, t. VIII, p. 341: "mot de citoyen annonce une corrélation avec les autres citoyens, et cette corrélation engendre les devoirs".

49 Cf. $A P$, t. VIII, p. 341.

50 Sobre el concepto de horizonte de expectativa, cf. R. Koselleck, "«Espacio de experiencia» y «horizonte de expectativa». Dos categorías históricas”, en Futuro pasado. Para una semántica de los tiempos históricos, Barcelona, Paidós, [1979] 1993, pp. 333-357. 
"una vez fijadas las premisas, no se puede detener las consecuencias" 51 . En efecto, el carácter performativo que la Declaración promovió rápidamente la apropiación y diseminación de los principios allí enunciados ${ }^{52}$. "La formulación universalista y abstracta de los derechos-observa Laurence Cornu-es precisamente lo que infunde y autoriza a quienes estaban injustamente apartados, a apelar a esos mismos derechos para ser reconocidos y protegidos por ellos"53. Mediante la consagración del principio de igualdad natural de derechos, la Declaración dinamizó la lucha por el reconocimiento, produciendo, en varios casos, un efecto cascada sobre el campo del derecho. Ciertamente, como señala Lynn Hunt, "Los acontecimientos empujaron a los diputados a reconocer su aplicabilidad a lugares y grupos que en un principio habían quedado al margen" ${ }^{54}$. Bajo la lógica del principio de igualdad natural de derechos, los no-católicos por ejemplo, serían admitidos en todos los puestos civiles y militares hacia fines de $1789^{55}$. Bajo el peso de la misma lógica, los judíos, excluidos originalmente de aquellos derechos, los obtendrían a fines de $1791^{56}$. De hecho, tras la caída de Robespierre, durante las deliberaciones suscitadas por la necesidad de elaborar una nueva declaración de derechos, los termidorianos habrían de renegar de la redacción de 1789, pues, según su opinión, la forma en la que había sido enunciado su primer artículo fomentaba la rebelión de aquellos que no gozaban plenamente de la igualdad de derechos ${ }^{57}$.

El profundo impacto político y moral que produjo la Declaración terminó por otorgarle un valor cuasi sagrado entre los franceses, concediéndole de ese modo una autonomía propia, incluso respecto de la voluntad de sus propios autores. Originalmente, el Comité de constitución había sugerido que la declaración de derechos no fuese adoptada ni publicada antes de terminar la redacción de la carta constitucional, ya que así era posible poner en correlación los principios enunciados en ambos documentos jurídicos.

El comité ha creído—señaló Mounier-que sería conveniente, para recordar el fin de nuestra constitución, el precederla con una declaración de los derechos de los hombres, pero colocándola, en forma de preámbulo, por encima de los artículos constitucionales, y no publicándola separadamente. El comité ha pensado que esta última opción presentaría poca utilidad y podría traer inconvenientes. Las ideas arbitrarias

51 M. Gauchet, op. cit., p. 199: "les prémisses une fois posées, on n'arrête pas les conséquences".

52 "El verbo «declarar»- - señala Laurence Cornu—es un «performativo», pues no designa un acto anterior a su enunciación, sino que él mismo ese acto" (L. Cornu, op cit., p. 174: "Le verbe «déclarer» est un «performatif»: il ne désigne pas un acte antérieur à son énonciation, il est lui-même cet acte"). Sobre el sentido otorgado al concepto de diseminación, cf. Pablo Facundo Escalante, "Pensando la democratización de los lenguajes y conceptos políticos desde una perspectiva derrideana", Conceptos Históricos, n 3, 2016, pp. 132-151, URL: http:// www.unsam.edu.ar/ojs/index.php/conhist/article/view/206.

53 L. Cornu, op. cit., p. 192: "La formulation universaliste et abstraite des droits est précisément ce qui incite et autorise ceux qui étaient injustement écartés à se réclamer de ces droits mêmes pour y être reconnus et protégés".

54 L. Hunt, op. cit., p. 168.

55 Cf. "Décret qui déclare les non-catholiques admissibles à tous les emplois civils et militaires (24 de diciembre de 1789)", en J.-B. Duvergier (ed.), Collection complète des Lois, Décrets, Ordonnances, Réglemens, Avis du Conseil-d'État (De 1788 à 1830 inclusivement, par ordre chronologique), t. I, Paris, A. Guyot et Scribe, 1834, p. 89. Con el término "no-católicos" se entendía a los cristianos reformados y, en particular, a los hugonotes.

56 Cf. "Décret relatif aux Juifs qui prêteront le serment civique (27 de septiembre de 1791)", en J.-B. Duvergier (ed.), op. cit., t. III, pp. 374-375.

57 Cf. M. Gauchet, op. cit., pp. 302-307. 
y filosóficas, si no son acompañadas por sus consecuencias, permitirán extraer otras, aparte de las que serían admitidas por la Asamblea. Al no adoptar definitivamente la declaración de derechos hasta el momento en que se haya acabado el examen de todos los artículos de la constitución, se tendría la ventaja de combinar con mayor exactitud todo lo que debe entrar en la exposición de principios y ser aceptado como su consecuencia. Esta declaración debería ser corta, simple y precisa. Es, pues, de la declaración de derechos, considerada como preámbulo de la constitución, que la Asamblea debe ocuparse primero, sin adoptarla definitivamente ${ }^{58}$.

Los diputados terminarían desoyendo las recomendaciones del Comité de constitución. Los diecisiete artículos decretados entre el 20 y el 26 de agosto serían adoptados y publicados inmediatamente. Con todo, la Asamblea conservaría la idea de revisar y modificar la Declaración una vez que hubiese acabado de redactar la Constitución. En agosto de 1791, en el marco del proceso de revisión de los artículos constitucionales, habría de proponerse efectivamente que se hiciera lo mismo con aquellos que correspondían a la declaración de derechos. En aquella ocasión, sin embargo, Thouret, en nombre de los Comités de constitución y de revisión, realizaría una férrea defensa de la redacción de 1789:

La declaración de los derechos del hombre y del ciudadano está a la cabeza de nuestro trabajo. Allí se encuentra exactamente tal y como ha sido decretada. Los comités no han creído que les estuviese permitido proponeros el realizar en ella algún cambio. Creen inclusive que no sería bueno que se realizara cambio alguno. Esta declaración ha adquirido, de cierta forma, un carácter sagrado y religioso. Se ha convertido, tras dos años, en el símbolo de todos los franceses: ha sido impresa en todos los formatos; se encuentra en afiches en todos los lugares públicos y hasta en las viviendas de los habitantes del campo; ha servido y sirve para que los niños aprendan a leer. Sería muy peligroso establecer, en paralelo a esta declaración, una segunda declaración, no solamente diferente en el fondo de las cosas, sino diferente inclusive en la redacción, pues ya no se podría distinguir cuál sería la verdadera declaración de los derechos del hombre y del ciudadano. Creemos que como ella contiene todos los principios elementales, como contiene todos los gérmenes, todas las consecuencias útiles que se pueden desear y todos los estímulos ventajosos para el estado social y para los derechos de los individuos y de la sociedad, creemos, pues, que es absolutamente inútil realizar cambio alguno a esta declaración, y, como decía, sería peligroso hacerlo ${ }^{59}$.

58 Mounier, 9 de julio de 1789, en $A P$, t. VIII, p. 216: "Le comité a cru qu'il serait convenable, pour rappeler le but de notre constitution, de la faire précéder par une déclaration des droits des hommes, mais de la placer, en forme de préambule, au-dessus des articles constitutionnels, et non de la faire paraître séparément. Le comité a pensé que ce dernier parti présenterait peu d'utilité, et pourrait avoir des inconvénients; que des idées arbitraires et philosophiques, si elles n'étaient accompagnées des conséquences, permettraient d'en supposer d'autres que celles qui seront admises par l'Assemblée; qu'en n'arrêtant pas définitivement la déclaration des droits jusqu'au moment où l'on aura achevé l'examen de tous les articles de la constitution, on aurait l'avantage de combiner plus exactement tout ce qui doit entrer dans l'exposé des principes, et être accepté comme conséquence. Cette déclaration devrait être courte, simple, et précise. C'est donc de la déclaration des droits, considérée comme préambule de la constitution, que l'Assemblée doit d'abord s'occuper, sans l'arrêter définitivement".

59 Thouret, 8 de agosto de 1791, en AP, t. XXIX, pp. 266-267: "La déclaration des droits de l'homme et du citoyen est en tête de notre travail. Elle y est exactement telle qu'elle a été décrétée. Les comités n'ont pas cru qu'il leur fût permis de vous proposer d'y faire aucun changement. Ils croient même qu'il ne serait pas bon qu'il y 
Claramente, para 1791, la Declaración se había convertido en el catecismo nacional, tal y como lo había demandado Barnave en $1789^{60}$. Los principios allí enunciados la habían convertido en un objeto de culto, inaccesible a la voluntad de sus propios creadores.

\section{Los límites del reconocimiento}

Dos factores se interpusieron rápidamente en el camino que conducía a la plena aplicación de los grandilocuentes principios morales enunciados en la Declaración. En efecto, el miedo al desbordamiento social y la propia práctica de la política pronto obligaron a los diputados a revisar aquello que habían postulado con tanto optimismo en esa suerte de tiempo suspendido que se había generado entre el 18 y el 26 de agosto de 1789.

Posiblemente-observa Timothy Tackett-, la inmensa mayoría coincidía en los objetivos generales de «libertad» e «igualdad» y los asumió con la convicción y el entusiasmo propios del converso. Sin embargo, aplicar estos principios en el seno de una sociedad muy poco acostumbrada a la realidad de tales valores, fijar los límites de la libertad, los de la igualdad y reconstruir un nuevo régimen partiendo de las ruinas del antiguo supondría, en conjunto, un reto extraordinario ${ }^{61}$.

La reclusión en el palacio de Versalles tuvo un efecto profiláctico sobre los diputados, preservando su optimismo político al mantenerlos alejados de los episodios de violencia que fueron suscitados por la propia Revolución. En efecto, los violentos acontecimientos que tuvieron lugar en la capital y en las provincias a lo largo del mes de julio sólo llegaron a ellos a través de los periódicos y los rumores. Sin embargo, en la madrugada del 6 de octubre, una multitud proveniente de París y compuesta mayoritariamente por mujeres irrumpió con violencia en el palacio de Versalles, atacando y masacrando a varios de los guardias que se encontraban allí apostados. La calma sólo retornaría gracias a la intervención de La Fayette y la Guardia nacional, y tras la promesa de Luis XVI de abastecer de pan a París, consentir los decretos de agosto - entre ellos la Declaración de derechos- y mudar su residencia al palacio de las Tullerías, ubicado en el centro de aquella ciudad.

fût fait aucun changement. Cette déclaration a, en quelque sorte, acquis un caractère sacré et religieux. Elle est devenue depuis deux ans le symbole de tous les Français, elle est imprimée dans tous les formats; elle se trouve en placards dans tous les lieux publics et jusque dans les habitations des habitants des campagnes; elle a servi et sert à apprendre à lire aux enfants. Il serait très dangereux d'établir en parallèle de cette déclaration, une seconde déclaration différente, non seulement différente par le fond des choses qu'elle pourrait contenir, mais même différente en rédaction. On ne connaitrait plus qu'elle est la véritable déclaration des droits de l'homme et du citoyen. Nous croyons donc que, comme elle contient tous les principes élémentaires, comme elle contient tous les germes, toutes les conséquences utiles qu'on peut désirer et toutes les inductions avantageuses à l'état social et aux droits des individus et de la société, nous croyons donc qu'il est absolument inutile de faire aucun changement a cette déclaration, et comme je disais, il serait dangereux de le faire".

60 "Creo—aseguró Barnave-que es indispensable el colocar a la cabeza de la constitución una declaración de los derechos que el hombre debe gozar. Es preciso que sea simple, al alcance de todas las mentes y que se vuelva el catecismo nacional" (Barnave, $1^{\circ}$ de agosto de 1789, en $A P$, t. VIII, p. 322: "Je crois qu'il est indispensable de mettre à la tête de la constitution une déclaration des droits dont l'homme doit jouir. Il faut qu'elle soit simple, à portée de tous les esprits, et qu'elle devienne le catéchisme national').

${ }_{61}$ T. Tackett, El Terror en la Revolución francesa, Barcelona, Pasado \& Presente, 2015, p. 15. 
La impresión que dejaron las jornadas de octubre sobre los diputados fue más que traumática. En la mayoría de casos, se trató de su primer contacto directo con la violencia popular ${ }^{62}$. De hecho, una de las consecuencias inmediatas de aquel episodio fue la elaboración y sanción de la ley marcial ${ }^{63}$. La Asamblea comenzó así su viraje hacia posiciones más moderadas o directamente conservadoras. Con todo, esto aparentemente no representó ninguna una contradicción para los diputados. En efecto, dos meses antes, en medio de los debates sobre la Declaración, Duport, futuro miembro del moderado Club de los feuillants, había señalado la necesidad de limitar los derechos que debían incluirse en ella en términos más que sugerentes: "El objeto de una declaración es entonces el de incluir todos los derechos, cualquiera que éstos sean. ¿Qué importa que sean contrarios a la Constitución? La declaración es para establecerlos, la Constitución es para modificarlos y circunscribirlos" ${ }^{\text {"64 }}$. Como observa Keith Michael Baker, "El temor por el desorden social requería que las implicaciones potencialmente subversivas de los derechos fueran limitadas por la ley" ${ }^{65}$. Incluso un pensador progresista como Condorcet llegaría a reconocer por aquel entonces que las expectativas a las que daba lugar el lenguaje utilizado en la Declaración comportaban una serie de peligros que sólo podrían ser prevenidos "exponiendo, por ejemplo, en qué sentido expresiones como la utilidad común o el mantenimiento del orden público deben ser entendidas para que las leyes estén siempre conformes al derecho y a la justicia" ${ }^{6}$.

En su traslado de Versalles a París, Luis XVI fue acompañado por la propia Asamblea, que mudó su sala de reuniones al picadero [manège], un edificio próximo al palacio de las Tullerías. A partir de entonces, los diputados estuvieron en contacto cotidiano con el constante descontento social que caracterizaba a la capital. Considerando la repugnancia que los diputados expresaban por todo tipo de violencia en general y por la violencia popular en particular ${ }^{67}$, no es coincidencia que los dos grupos sociales a los que la Constitución terminó por privar de derechos políticos fueron precisamente las mujeres - autoras de las jornadas de octubre- y los sectores de menores ingresos - acostumbrados a manifestar de forma violenta su descontento-. En opinión de los diputados, ambos grupos no poseían aún la educación necesaria para hacer un ejercicio activo de la ciudadanía. Tal y como observa Shanti Marie Singham, incluso las mujeres que participaban activamente en las reuniones del Círculo social, como Mme. Roland, coincidieron en aquella apreciación, asegurando que la educación era un prerrequisito indispensable para la emancipación femenina ${ }^{68}$. Con todo, como lo

62 Cf. ibidem, pp. 82-88.

${ }^{63}$ Cf. "Décret contre les attroupements, ou loi martiale (21 de octubre de 1789)", en J.-P. Duvergier (ed.), op. cit., t. I, pp. 52-53.

${ }^{64}$ Duport, 18 de agosto de 1789, en $A P$, t. VIII, p. 451: “L'objet d'une déclaration est donc de comprendre tous les droits quelconques. Qu'importe qu'ils soient contraires à la Constitution? La déclaration est pour les établir, la Constitution es pour les modifier et les circonscrire".

${ }^{65}$ K. M. Baker, "The Idea of a Declaration of Rights", op. cit., p. 192: "Fear of social disorder required that the subversive potential implications of rights be limited by the law".

66 N. de Condorcet, "Réflexions sur ce qui a été fait, et sur ce qui reste à faire (1789)", en A. Condorcet O'Connor y Fr. Arago (eds.), Euvres de Condorcet, t. IX, Paris, Firmin Didot Frères, 1847, pp. 441-468, aquí p. 449: “en exposant, par exemple, dans quel sens ces expressions, l'utilité commune, le maintien de l'ordre public, doivent être entendues pour que les lois soient toujours conformes au droit et à la justice".

${ }^{67}$ Cf. T. Tackett, op. cit., pp. 45-52

68 Cf. Sh. M. Singham, "Betwixt Cattle and Men. Jews, Blacks, and Women, and the Declaration of the Rights of Man”, en D. Van Kley (ed.), op. cit., pp. 114-153. 
señaló Barnave, aquella privación de derechos políticos no tenía por qué entenderse como una transgresión de los principios establecidos en la Declaración:

Todos los que han combatido la opinión de los comités han llegado a este error fundamental en sus razonamientos; han confundido el gobierno democrático y el gobierno representativo: es por ello que pudieron confundir los derechos del pueblo con la cualidad de electores, que no es más que una función pública a la cual nadie tiene derecho, sino que la sociedad la concede, así como le prescribe su interés ${ }^{69}$.

Entendido en esos términos, el sufragio no debía ser considerado como un derecho, sino más bien como una función pública. En consecuencia, la exclusión de ciertos grupos sociales teóricamente incapacitados para ejercerla se fundaba, en última instancia, sobre la propia utilidad común, tal y como lo establecía la propia Declaración en su artículo primero: "Los hombres nacen y permanecen libres e iguales en derechos. Las distinciones sociales no pueden estar fundadas más que sobre la utilidad común"70.

La práctica de la política, por su parte, obligó a los diputados a transigir con sus principios en función de otros imperativos o intereses políticos. Por ejemplo, la lógica igualitaria desplegada por la Declaración produjo un enorme descontento entre los grandes propietarios de esclavos que residían en las colonias francesas. Así, ante la amenaza de independencia realizada por los esclavistas, el 8 de marzo de 1790 la Asamblea emitió un decreto por el cual se establecía que cada colonia tendría la potestad de decidir si se plegaba o no a la futura Constitución y, por consiguiente, a la Declaración de los derechos del hombre que habría de encabezarla ${ }^{71}$. Esta cuestión no hace más que poner en evidencia que era imposible reducir la política a la moral, muy a pesar de las buenas intenciones que poseían los diputados. Ciertamente, la esclavitud había sido un tema que los había preocupado desde un primer momento. Esta preocupación se puso de manifiesto, por ejemplo, en los sucesivos proyectos de Constitución presentados en la Asamblea. Uno de ellos, presentado el 27 de julio de 1789, establecía lo siguiente:

Siendo Francia una tierra libre, la esclavitud no puede ser tolerada ahí, y cualquier esclavo queda emancipado, de pleno derecho, desde el momento en el que entra en Francia. Los procedimientos introducidos para eludir esta regla serán inútiles en el futuro, y, de ahora en adelante, ningún pretexto podrá oponerse a la libertad del esclavo ${ }^{72}$.

69 Barnave, 11 de agosto de 1791, en $A P$, t. XXIX, p. 366: "Tous ceux qui ont combattu l'opinion des comités se sont rencontrés dans cette erreur fondamentale de leurs moyens; ils ont confondu le gouvernement démocratique et le gouvernement représentatif: c'est pour cela qu'ils ont pu confondre avec les droits du peuple la qualité d'électeurs, qui n'est qu'une fonction publique à laquelle personne n'a droit, que la société dispense ainsi que le lui prescrit son intérêt".

70 Declaración de los derechos del hombre y del ciudadano de 1789, art. 1.

71 Cf. "Décret qui autorise les colonies à faire connaître leur vœu sur la constitution, la législation et l'administration qui leur conviennent (8 de marzo de 1790)", en J.-B. Duvergier (ed.), op. cit., t. I, p. 113.

72 "Projet contenant les premiers articles de la constitution (1789)", art. 8, en AP, t. VIII, pp. 285-287, aquí p. 286: "La France étant une terre libre, l'esclavage ne peut y être toléré, et tout esclave est affranchi, de plein droit, dès le moment où il est entré en France. Les formalités introduites pour éluder cette règle seront inutiles à l'avenir, et aucun prétexte ne pourra désormais s'opposer à la liberté de l'esclave". 
Otro proyecto, presentado el 12 de agosto, proponía la supresión de la esclavitud no sólo en el hexágono, sino también en cualquier territorio en poder de los franceses: "Todo esclavo recupera su libertad entrando en las tierras de la dominación francesa"73. En este último caso, merece destacarse, además, el uso del término "recuperar", el cual implica obtener de nuevo algo que se poseía antes y que, por algún motivo, se había perdido; un término en perfecta sintonía con los principios del futuro primer artículo de la Declaración.

La postura adoptada frente a la esclavitud en las colonias revela, en última instancia, la hipocresía de la crítica ilustrada respecto a la propia política, en la medida en que demandó la subordinación de esta última a la moral siempre que se halló fuera del aparato de gobierno, pero sólo cuando se hizo con el ejercicio de la soberanía logró comprender que era preciso incluir también otras variables en la ecuación ${ }^{74}$. En efecto, enfrentados a la posibilidad de perder las colonias - cuyo valor se expresaba no sólo en términos económicos, sino también en términos geopolíticos-, los diputados rápidamente dejaron de lado los principios de la Declaración, subordinando la moral a la política.

$* * *$

En su origen ilustrado y optimista, la Declaración fue concebida para operar como la brújula moral que debía guiar en todo momento a la política. A pesar de los obstáculos que en su momento impidieron la plena aplicación de los principios enunciados en ella, es innegable que su insistencia en la igualdad natural de derechos mediante el uso de un lenguaje abstracto y universalista, inauguró una lógica de reconocimiento recíproco que ha contribuido desde entonces a desplazar, de forma incesante, los límites de aquello que se considera moralmente injusto, aun si luego tales injusticias no pudieron (o no quisieron) ser reparadas por la propia política.

73 "Charte contenant la constitution française dans ses objets fondamentaux (1789)", art. 19, en $A P$, t. VIII, pp. 400-403, aquí p. 401: "Tout esclave reprend sa liberté entrant dans les terres de la domination française".

74 Cf. R. Koselleck, Crítica y crisis. Un estudio sobre la patogénesis del mundo burgués, Madrid, Trotta/Universidad Autónoma de Madrid, [1959] 2007. 